\title{
Spectral Observations of the Diffuse FUV Background with DUVE (the Diffuse UV Experiment)
}

\author{
Eric J. Korpela and Stuart Bowyer
}

Space Sciences Laboratory, University of California, Berkeley, CA 94720

\begin{abstract}
We present results from a search for FUV emission from the diffuse ISM conducted with an orbital FUV spectrometer, DUVE, which was launched in July, 1992. The DUVE spectrometer, which covers the band from $950 \AA$ to 1080 $\AA$ with $3.2 \AA$ resolution, observed a region of low neutral hydrogen column density near the south galactic pole for a total effective integration time of 1583 seconds. the only emission line detected was a geocoronal hydrogen line at $1025 \AA$. We were able to place upper limits to several emission features that provide constraints to interstellar plasma parameters. We were also able to place continuum limits in this band. We use these upper limits to place constraints upon the emission measure vs. temperature distribution of this gas using an isothermal Landini and Fossi model.
\end{abstract}

\section{Introduction}

Since the prediction of the existence of hot gas in the interstellar medium by Spitzer (1956) and its subsequent detection by Bowyer, Field and Mack (1968) in soft X-rays, a variety of models have been developed which attempt to explain the source of this gas, and how it evolves over time. As more data has become available, especially observations of absorption from high stage ions, these models have evolved to fit the data (Slavin and Cox, 1993). Observations of FUV emission are key to differentiating between these models.

\section{The Instrument and Calibration}

We have designed an instrument capable of measuring the important Ovi $\lambda \lambda$ 1032,1038 emission from the diffuse interstellar medium. The instrument's small bandpass $(150 \AA)$ around the Ovi lines also includes the potentially important CiII $\lambda$ 977, NiII $\lambda$ 991, and CII $\lambda 1037$ lines. The instrument, designated DUVE, The Diffuse Ultraviolet Experiment, is based on a spectrometer developed by Edelstein and Bowyer (1993). That instrument's capability for studying diffuse radiation was substantially improved. The basic design is a two stage spectrometer. A wire-grid collimator with a field of view of $2^{\circ} \times 7^{\circ}$ in conjunction with a diffraction grating in Wadsworth configuration and an exit slit serve to limit the instrument bandpass from 950 to $1080 \AA$. The second stage of the spectrometer is a dispersion stage. Light entering through 
the slit strikes a holographically corrected diffraction grating in a Rowland circle configuration. The second order diffracted light from the grating is focused onto a microchannel plate detector. The high spectral resolution of the dispersion stage $(3.5 \AA)$ allows the OVI doublet to be separated from the much brighter Ly $\beta \lambda 1026$ airglow line. Extensive calibration of the DUVE instrument was carried out using the EUV/FUV calibration facilities at the Space Sciences Laboratory (Welsh et al. 1989). The DUVE instrument was launched as a secondary payload attached to the second stage of a Delta II 7925 vehicle on $24 \mathrm{July}, 1992$. A morning launch resulted in a near midnight apogee of $1460 \mathrm{~km}$ (750 nmi). The mission continued for $3 \times 10^{4}$ seconds, until the second stage telemetry battery was depleted.

\section{Observational Results}

The successful mission resulted in 19 images containing 4151 seconds of observation time. Half of the observation time was made with the shutter closed, for accurate calibrations of the instrument background. The shutter opened images were examined for stellar contamination, uncorrected data dropouts and instrument anomalies and the affected portions were masked off or otherwise corrected. Where no correction was possible, the images were discarded.

The sum of these images was corrected for image distortions and shutter open/closed time, and the shutter-closed background images were subtracted from the data. The resulting image was histogrammed and convolved with a line spread function that was determined during calibration.

The only spectral line that exceeds the $3 \sigma$ significance level is the geocoronal Ly $\beta \lambda 1025$ line. We have used the DUVE data to put upper limits to line emission in the band covered by the spectrometer assuming line widths of less than $3.5 \AA$. These line emission limits were determined by calculating the upper $90 \%$ statistical confidence level of the measured spectrum at each wavelength. These limits are shown in Table 1 . The best determinations of continuum level were $-1.07 \pm 0.69 \times 10^{3} \mathrm{ph} \mathrm{s}^{-1} \mathrm{~cm}^{-2} \mathrm{str}^{-1} \AA^{-1}$ between 977 and $1020 \AA$ and $-3.2 \pm 3.8 \times 10^{2} \mathrm{ph} \mathrm{s}^{-1} \mathrm{~cm}^{-2} \operatorname{str}^{-1} \AA^{-1}$ between 1028 and $1057 \AA$. These measurements allow us to place $2 \sigma$ upper limits in these ranges of 310 and $440 \mathrm{ph} \mathrm{s}^{-1} \mathrm{~cm}^{-2} \mathrm{str}^{-1} \AA^{-1}$ respectively.

\section{Isothermal Models}

The simplest (and most often used) model of emission from the warm and hot phases of the interstellar medium is that of an optically thin, isothermal, collisionally excited plasma. Although collisional ionization equilibrium (CIE) is unlikely to occur in interstellar gas, virtually all observational results are expressed in terms of CIE. We do the same for purposes of comparison. For the purposes of this determination we have used the CIE code of Landini 
Table 1. Upper limits to line emission placed by the DUVE data

\begin{tabular}{|crrr|}
\hline Species & $\lambda(\AA)$ & $I\left(\mathrm{ph} \mathrm{s}^{-1}\right.$ & $\left.\mathrm{cm}^{-2} \mathrm{str}^{-1}\right) I\left(\mathrm{erg} \mathrm{s}^{-1} \mathrm{~cm}^{-2} \mathrm{str}^{-1}\right)$ \\
\hline \hline $\mathrm{HI}^{1}$ & 972 & $\leq 7.4 \times 10^{4}$ & $\leq 1.5 \times 10^{-6}$ \\
$\mathrm{CIII}^{2}$ & 977 & $\leq 4.0 \times 10^{4}$ & $\leq 8.1 \times 10^{-7}$ \\
$\mathrm{OI}^{1}$ & 989 & $\leq 6.1 \times 10^{3}$ & $\leq 1.2 \times 10^{-7}$ \\
$\mathrm{NIII}^{2}$ & 991 & $\leq 5.5 \times 10^{3}$ & $\leq 1.1 \times 10^{-7}$ \\
SiII $^{2}$ & 992 & $\leq 5.7 \times 10^{3}$ & $\leq 1.1 \times 10^{-7}$ \\
SiII & 996 & $\leq 1.6 \times 10^{4}$ & $\leq 3.2 \times 10^{-7}$ \\
NeVI & 1006 & $\leq 1.3 \times 10^{4}$ & $\leq 2.6 \times 10^{-7}$ \\
ArVI & 1008 & $\leq 1.0 \times 10^{4}$ & $\leq 2.0 \times 10^{-7}$ \\
$\mathrm{HI}^{1,3}$ & 1025 & $2.26 \pm 0.26 \times 10^{5}$ & $4.38 \pm 0.49 \times 10^{-6}$ \\
OVI $^{2,4}$ & 1032,1038 & $\leq 7.6 \times 10^{3}$ & $\leq 1.4 \times 10^{-7}$ \\
$\mathrm{CII}^{2}$ & 1037 & $\leq 3.9 \times 10^{3}$ & $\leq 7.4 \times 10^{-8}$ \\
ArI $^{1}$ & 1050 & $\leq 6.4 \times 10^{3}$ & $\leq 1.2 \times 10^{-7}$ \\
SiIV & 1067 & $\leq 2.9 \times 10^{4}$ & $\leq 5.4 \times 10^{-7}$ \\
SIV & 1070 & $\leq 5.1 \times 10^{4}$ & $\leq 9.5 \times 10^{-7}$ \\
\hline
\end{tabular}

${ }^{1}$ Anticipated airglow line

${ }^{2}$ Constrains state of hot interstellar medium

${ }^{3}$ Detected at $37 \sigma$.

4 This limit is total emission from the doublet based upon joint statistics by assuming $\frac{I(1032)}{I(1038)}=2$. Upper limits for the individual components of the doublet are $I(1032) \leq 5400 \mathrm{ph} \mathrm{s}^{-1} \mathrm{~cm}^{-2} \mathrm{str}^{-1}$ and $I(1038) \leq 4400 \mathrm{ph} \mathrm{s}^{-1} \mathrm{~cm}^{-2} \mathrm{str}^{-1}$.

and Monsignori-Fossi (1990). Using the upper limits obtained by the DUVE instrument, we can place upper limits to the emission measure of isothermal plasma models for the interstellar medium (Fig.1). The emission measure of the interstellar medium is constrained by five emission lines in this band:

1. MgII $\lambda 1027$, which peaks at about $1.5 \times 10^{4} \mathrm{~K}$, constrains the emission measure at temperatures below $2.0 \times 10^{4} \mathrm{~K}$. Because of the nearby interference from Ly $\beta 1025$ emission, the observational limit obtained is very high.

2. CII $\lambda$ 1037, which peaks at a temperature of about $5 \times 10^{4} \mathrm{~K}$, provides the best constraint for temperatures between $2.0 \times 10^{4}$ and $5.1 \times 10^{4} \mathrm{~K}$. Because our simple model does not include photoionization, this may understate the constraint, as CII is expected to be produced by photoionization due to FUV radiation from stars.

3. CiI $\lambda 977$, which peaks at $8.4 \times 10^{4} \mathrm{~K}$, provides the best constraint between $5.1 \times 10^{4}$ and $1.0 \times 10^{5} \mathrm{~K}$.

4. NiII $\lambda$ 991, which peaks at $1 \times 10^{5} \mathrm{~K}$, constrains the emission measure between $1.0 \times 10^{5}$ and $1.8 \times 10^{5} \mathrm{~K}$.

5. Ovi $\lambda \lambda 1032,1038$, which peaks at $2.8 \times 10^{5} \mathrm{~K}$, provides upper limits to the emission measure above $1.8 \times 10^{5} \mathrm{~K}$. 


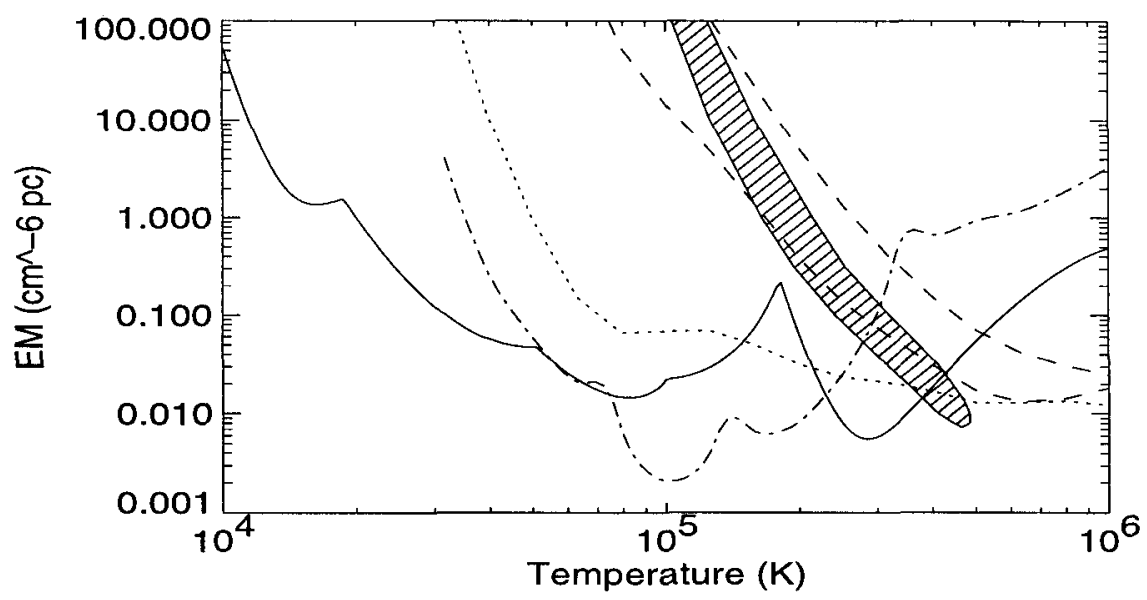

Fig. 1. Upper limit local emission measures derived from this work are shown as a solid line. The dashed lines represent $\mathrm{X}$-ray emission measure limits derived for Wisconsin $\mathrm{B}$ and $\mathrm{C}$ band rocket borne observations in this direction (McCammon et al.1983). The dot-dashed line represents emission measure limits determined from UVX (1400 to $1800 \AA$ ) observations by Martin and Bowyer (1990). The dotted line represents upper limits determined from EUVE observations by Jelinsky et al (1995). The hatched area is the parameter space cited by Paresce and Stern (1981) as being the allowed region to create observed broadband EUV and Soft X-ray emission.

A more detailed discussion of these results may be found in Korpela et al. (1997).

\section{References}

Bowyer, C. S., Field, G. B., \& Mack, J. E., Nature, 1968, 351, 32.

Dixon, W. V., Davidsen, A. F. \& Ferguson, H. C., 1996, ApJ, 465, 288.

Edelstein, J. \& Bowyer, S., 1993, AdSpR, v13, n12, 307.

Korpela, E., Bowyer, S. and Edelstein, J., 1997, Submitted to ApJ.

Jelinsky, P., Vallerga, J. V. \& Edelstein, J., 1995, ApJ, 442, 653.

Landini, M., \& Monsignori Fossi, B. C., 1990, A\&AS, 82, 229.

Martin, C. \& Bowyer, S., 1990, ApJ, 350, 242.

McCammon, D., Burrows, D. N., Sanders, W. T., Kraushaar, W. L., 1983, ApJ, 269, 107.

Paresce, F. \& Stern, R., 1981, ApJ, 247, 89.

Slavin, J. and Cox, D., ApJ, 417, 187, 1993.

Spitzer, L., 1956, ApJ, 124, 20.

Welsh, B., Vallerga, J. V., Jelinsky, P., Vedder, P. W., Bowyer, S., \& Malina R. F., 1989, Proc. SPIE, 1160, 554. 